\title{
Pattern Formation Near Onset of a Convecting Fluid In an Annulus
}

\author{
Berk Sensoy and Henry Greenside* \\ Department of Physics \\ Duke University, Durham, NC 27r08-0305
}

(January 25, 2001)

\begin{abstract}
Numerical simulations of the time-dependent Swift-Hohenberg equation are used to test predictions of Cross [Phys. Rev. A 25:1065-1076 (1982)] that Rayleigh-Bénard convection in the form of straight rolls or of an array of dislocations may be observed in an annular domain depending on the values of inner radius $r_{1}$, outer radius $r_{2}$, reduced Rayleigh number $\epsilon$, and the initial state. As $r_{1}$ is decreased for a fixed $r_{2}$ and for different choices of $\epsilon$ and initial states, we find that there are indeed ranges of these parameters for which the predictions of Cross are qualitatively correct. However, when the radius difference $r_{2}-r_{1}$ becomes larger than a few roll diameters, a new pattern is observed consisting of stripe domains separated by radially-oriented grain boundaries. The relative stabilities of the various patterns are compared by evaluating their Lypunov functional densities.
\end{abstract}

05.70.Ln, 47.54.+r 47.27.Te

\section{INTRODUCTION}

A central question of nonequilibrium physics is pattern formation, why only certain stationary patterns in a nonequilibrium, homogeneous, and continuous medium are observed for fixed parameters and boundaries. In this paper, we investigate a particular aspect of this question, namely how does pattern formation depend on the shape of the lateral boundaries. We do so by numerically integrating a two-dimensional model of a three-dimensional convecting fluidthe Swift-Hohenberg equation [1,2] - in an annular domain as a function of the inner and outer radii of the annulus and of the reduced Rayleigh number $\epsilon$ (which measures how strongly the fluid is driven out of equilibrium). Theory predicts that, as parameters are varied, two kinds of patterns may be observed, a defect-free straight roll texture or an array of dislocations [3]. The results we present below confirm some of these predictions and also predict a new state of radially oriented grain boundaries if the radial width becomes sufficiently large. Future Rayleigh-Bénard experiments with a large Prandtl number fluid near onset in an annular domain may be able to confirm these results.

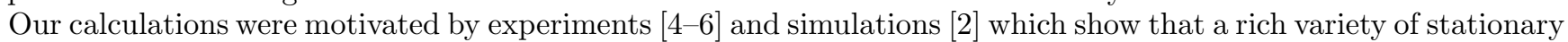
patterns are observed just above the onset of Rayleigh-Bénard convection in the form of stripes (locally periodic convection rolls) disordered by topological defects such as dislocations, focus singularities, and grain boundaries. (Section VIII of the review article by Cross and Hohenberg [f] discusses many experimental and theoretical results for Rayleigh-Bénard convection.) The occurrence of defects can be qualitatively understood as a consequence of two competing effects. First, near onset the local wave numbers of the convection rolls are constrained by the Busse stability balloon 810 to lie in a narrow range about the critical wave number $q_{c}$. Second, in the absence of horizontal thermal gradients, convection rolls are observed to evolve so as to approach the lateral boundaries at an approximately normal angle [4]. For the most commonly used experimental geometries of cylinders or boxes, these two constraints conflict with one another since it is not possible for the rolls to be everywhere normal to the sidewalls and to wiggle sinusoidally on a single length scale. This conflict can be partially resolved by the formation of topological defects which allow a local change in wave vector.

An insightful analysis of this conflict between lateral boundary conditions and local wave number was given by Cross [3], who observed that sufficiently close to the onset of convection, the pattern formation was governed by a Lyapunov functional $\mathcal{L}$ that decreases monotonically along any given orbit and that was bounded from below. Such a functional $\mathcal{L}$ implies that all initial conditions relax asymptotically to some stationary pattern and also provides a way to order different stationary patterns by their relative stability [3]. In the double limit

$$
\epsilon \rightarrow 0^{+} \quad \text { and } \quad \epsilon^{1 / 2} \Gamma \rightarrow \infty
$$

where $\epsilon$ is proportional to the reduced Rayleigh number $\left(R-R_{c}\right) / R_{c}$ and where $\Gamma$ is the aspect ratio (ratio of the largest lateral dimension to the fluid depth), Cross showed that the Lyapunov functional $\mathcal{L}$ could be decomposed into a sum of three terms, a surface term associated with the lateral boundaries, a bulk term for the fluid far from the boundaries, and a defect term which depends on the particular defects present. By comparing these three terms with one another for various geometries in the limit Eq. (1), Cross was able to explain rather subtle phenomena such as why a pattern of straight parallel rolls should be observed sufficiently close to onset in a large cylindrical or annular 
domain, as opposed to a pattern of concentric rolls with the same axisymmetry as the domain. However, a careful comparison of Cross's calculations with experiment [4] or with numerical simulation has not been carried out and it remains unclear to what level of accuracy the limits in Eq. (11) need to be satisfied for Cross's asymptotic analysis to hold. Experiments and numerical simulations can also help to determine when higher-order terms in the amplitude expansion destroy the property of variational dynamics, leading to a sustained oscillatory or chaotic time dependence.

Of particular interest to us are Cross's predictions about possible patterns in an annular domain. For this geometry, a fundamental frustration can arise if the rolls are normal to both the inner and outer boundaries since it is then not possible for the local wave number to remain close to the critical value $q_{c}$ as a function of radius in the limit of a large radial difference $r_{2}-r_{1}$. Cross predicted two possibilities that are illustrated in Fig. 7 of his 1982 paper [3]. One was that sufficiently close to onset, the bulk term in the Lyapunov functional would dominate, which would favor straight rolls that extend throughout the annular cell. Wave number frustration and defects are then avoided since the rolls are not normal to the sidewalls. As a second possibility, Cross predicted that the surface term would be more important further above onset in which case the rolls would orient normal to the inner and outer boundaries. An average wave number close to the critical value $q_{c}$ could then be arranged by introducing an array of dislocations (an example is shown below in Fig. 11). To our knowledge, these predictions have not been tested experimentally or numerically.

In this paper, we test these predictions by numerical simulations of the two-dimensional time-dependent SwiftHohenberg equation [1] in an annular geometry as a simple opportunity to explore how lateral boundaries affect pattern formation. The Swift-Hohenberg equation is a widely-studied rotationally invariant model that undergoes a supercritical bifurcation from a zero field state to a stripe state, corresponding to the supercritical bifurcation of a conducting motionless fluid to finite-amplitude three-dimensional convection rolls. Sufficiently close to the onset of convection, the long-wavelength slow-timescale dynamics of the Swift-Hohenberg equation obeys the same amplitude equation as the one obtained from the three-dimensional Boussinesq equations that describe a convecting fluid quantitatively [7]. The linear stability boundaries of the Swift-Hohenberg equation are also similar to that of the Boussinesq equations near onset [2, 11. Numerical integrations of the Swift-Hohenberg equation in periodic, rectangular, and cylindrical geometries [2, 12, 13] have shown that its solutions are often in semi-quantitative agreement with experiments sufficiently close to onset. It is then often useful to explore some questions of pattern formation first with Eq. (2) and later make more careful studies by experiment or by more expensive simulations of the Boussinesq equations.

Using a new time-integration code that we have developed to integrate the Swift-Hohenberg equation efficiently and accurately in large annular domains [14], we have calculated transient solutions and their asymptotic stationary patterns for different initial states and for different values of the parameters $r_{1}, r_{2}, \epsilon$. As we describe below, our results confirm parts of Cross's predictions but we also find that his analysis was incomplete in that a new state occurs when the radius difference $r_{2}-r_{1}$ becomes sufficiently large. This new state consists of patches of stripes separated by radially-oriented grain boundaries.

Using a modified version of the code, we have also integrated a Generalized Swift-Hohenberg (GSH) model [11, 15] to explore whether the wave number frustration caused by the annular geometry may lead to a sustained dynamics. The GSH model couples a second field, the vertical vorticity potential $\zeta(t, x, y)$, to Eq. (2) in such a way that the overall dynamics is no longer variational and sustained time-dependent solutions are possible 16 19. Our admittedly brief study suggests that a sustained dynamics exceeding 30 or more horizontal diffusion times $\left(\tau_{h}\right)$ seems to occur for $\Gamma>15$ while for $\Gamma<15$, long-lived transients were observed that decayed toward a time-independent state. However, further studies will be needed to determine more carefully whether any of the states are truly chaotic (what might appear to be chaotic states in a spatially extended system might instead be long-lived transients whose average decay time grows rapidly with the system size 201) and to characterize the dependence of the dynamics on aspect ratio and other parameters.

The rest of this paper is organized as follows. In Section II, we summarize details of the numerical method used to integrate the time-dependent Swift-Hohenberg equation in an annular geometry and to estimate the average Lyapunov functional $\langle\mathcal{L}\rangle$. In Section III, we discuss the results of our simulations and compare them to the asymptotic analysis of Cross and to the Busse linear stability balloon for the Swift-Hohenberg equation [1]. Finally, in Section IV, we summarize our main results. Further details are available in the undergraduate Physics thesis of the first author, on which this paper is based [14].

\section{METHODS}

In this section, we discuss some details of how the Swift-Hohenberg equation was integrated numerically in a large annular domain. The two-dimensional time-dependent Swift-Hohenberg equation [1, 2, ,7] 


$$
\partial_{t} \psi=\left[\epsilon-\left(\nabla^{2}+1\right)^{2}\right] \psi-\psi^{3},
$$

determines the time evolution of a real scalar field $\psi(t, r, \theta)$ in a two-dimensional spatial domain. Here $\partial_{t}=\partial / \partial t$ denotes the partial derivative with respect to time, $\nabla^{2}=\partial_{x}^{2}+\partial_{y}^{2}=\partial_{r}^{2}+r^{-1} \partial_{r}+r^{-2} \partial_{\theta}^{2}$ is the two-dimensional Laplacian operator, and the parameter $\epsilon$ is the bifurcation parameter, such that the zero state $\psi=0$ becomes linearly unstable at a critical value $\epsilon_{c}(\Gamma) \geq 0$. (As shown in Fig. 3 of Ref. [2], for rolls parallel to a wall this critical value for the boundary conditions Eq. (5) below goes to zero as $(2 / \Gamma)^{2}$ in the limit $\Gamma \rightarrow \infty$ so $\epsilon_{c}$ is tiny for $\Gamma>10$.) The relation of Eq. (2) to the amplitude equation of the Boussinesq equations shows [3] that, near onset (i.e., in the limit $\epsilon \rightarrow \epsilon_{c}^{+}$), the field $\psi$ is proportional to the temperature deviation $T\left(t, x, y, z_{0}\right)-T_{\text {linear }}\left(z_{0}\right)$ of the temperature field $T$ from its linear conducting profile $T_{\text {linear }}$, evaluated on the horizontal midplane $z=z_{0}$ of a three-dimensional convection cell. Positive and negative values of $\psi$ can therefore be interpreted as warmer (rising) and cooler (descending) regions respectively of a convecting fluid.

We solve Eq. (2) in polar coordinates $(r, \theta)$ on the annular domain

$$
r_{1} \leq r \leq r_{2}, \quad 0 \leq \theta<2 \pi,
$$

where the inner radius $r_{1}$ and outer radius $r_{2}$ are prescribed parameters. Since the scaling of parameters that lead to Eq. (2) normalizes the critical wave number to have the value $q_{c}=1$, the effective fluid depth for the Swift-Hohenberg equation is $d=(1 / 2)\left(2 \pi / q_{c}\right)=\pi$ and the aspect ratio $\Gamma$ of the annular cell is

$$
\Gamma=\frac{r_{2}-r_{1}}{\pi} .
$$

For boundary conditions, we require that the field $\psi$ and its normal derivative $\partial_{r} \psi$ vanish at each point of the inner boundary $r=r_{1}$ and at each point of the outer boundary $r=r_{2}$ :

$$
\psi\left(r_{i}, \theta\right)=0, \quad \text { and } \quad\left(\partial_{r} \psi\right)\left(r_{i}, \theta\right)=0, \quad i=1,2 .
$$

Although these conditions are consistent with the vanishing of the amplitude field $A=0$ as derived to lowest-order in an amplitude expansion in $\epsilon$ [3], they do not correspond to the correct boundary conditions of the amplitude $A$ further from onset. It is then perhaps best to consider the conditions Eq. (5) as convenient phenomenological conditions that numerous numerical calculations [2, 16] have shown to yield reasonable solutions when compared with large-aspectratio convection experiments near onset.

The numerical integration of Eq. (2) with boundary conditions Eq. (5) produces a discrete numerical solution $\psi_{i j k}$ at successive equally spaced times $t_{i}=i \Delta t$ that approximates the unknown analytical solution $\psi(t, r, \theta)$ on mesh points $\left(r_{j}, \theta_{k}\right)$ defined by

$$
\begin{aligned}
& r_{j}=r_{1}+j \Delta r, \quad 0 \leq j \leq N_{r}, \\
& \theta_{k}=k \Delta \theta, \quad 0 \leq k \leq N_{\theta} .
\end{aligned}
$$

Here $\Delta r=\left(r_{2}-r_{1}\right) / N_{r}$ and $\Delta \theta=2 \pi /\left(N_{\theta}+1\right)$ are the radial and angular resolutions, and the discrete solution converges to the unknown analytical solution at the mesh points in the limit of vanishing space-time resolution:

$$
\psi_{i j k} \rightarrow \psi\left(t_{i}, r_{j}, \theta_{k}\right) \quad \text { as } \quad \Delta t, \Delta r, \Delta \theta \rightarrow 0
$$

We discretized Eqs. (22) and (5) on the interior and boundary mesh points by approximating all spatial derivatives with second-order-accurate finite differences in the quantity $\psi_{i j k}$ as described by Bjørstad in Ref. [21]. For example, to approximate the biharmonic operator $\nabla^{4}$ to second-order accuracy at a given lattice point $\left(r_{j}, \theta_{k}\right)$, a linear combination of 13 lattice values involving up to second-nearest neighbors was used.

Since an explicit time-stepping method for Eq. (2) would impose a severe stability constraint of the form $\Delta t<$ $C \max \left(\Delta r^{4},(\Delta r \Delta \theta)^{4}\right)$ where $C$ is some constant 22], we chose to integrate Eq. (2) with a simple-to-implement operator-splitting method that integrates the nonlinear term explicitly and then the linear terms in Eq. (2) implicitly. The absence of spatial derivatives in the cubic nonlinear term then implies that the largest time step allowed by the algorithm is no longer bounded by some power of the spatial resolution, although it remains restricted by the overall stability of the time-splitting algorithm and by accuracy. By making runs with different temporal resolutions for a fixed fine spatial resolution, we found that a time step of $\Delta t=0.5$ provided a reasonable balance between accuracy and efficiency and we used this value for most of the integrations described in the next section. The code was sufficiently fast on a workstation using a $667-\mathrm{MHz}$ Alpha processor that we could integrate tens of horizontal thermal diffusion times (many multiples of $\Gamma^{2}$ ) for our largest $\Gamma$ domains in just a few days and so find approximately time-independent asymptotic patterns to good accuracy. 
The first step of our algorithm integrates the nonlinear piece $N[\psi]=-\psi^{3}$ explicitly, using a second-order-accurate Adams-Bashforth method:

$$
\psi^{*}=\psi_{i}+\frac{\Delta t}{2}\left(3 N\left[\psi_{i}\right]-N\left[\psi_{i-1}\right]\right) .
$$

This advances the known field field values $\psi_{i}$ at time $t_{i}$ (we temporarily suppress the spatial indices $i j$ to simplify the notation) to intermediate field values that we denote by $\psi^{*}$. The field values at time $t_{i+1}$ are then obtained by solving the linear pieces in Eq. (2) implicitly using a backward-Euler method with initial data $\psi^{*}$, leading to the equation

$$
\left(\nabla^{4}+2 \nabla^{2}+1+\frac{1}{\Delta t}-\epsilon\right) \psi_{i+1}=\frac{1}{\Delta t} \psi^{*},
$$

with the boundary conditions Eq. (5) imposed on $\psi_{i+1}$. This constant-coefficient generalized linear biharmonic problem can be solved efficiently with a second-order-accurate fast-direct method invented by Bjørstad [21, 23, 24] which has a nearly optimal computational complexity of $O\left(N_{r} N_{\theta} \log \left(N_{\theta}\right)\right)$. We used the public Fortran77 version of the annular Bjørstad solver available through netlib [25].

The input data for the computer code are then the parameters $\epsilon, r_{1}, r_{2}, N_{r}, N_{\theta}, \Delta t$, the total integration time $T$, and some initial discrete field $\psi_{0 j k}$ on the mesh Eq. (5) that satisfies the discrete form of the boundary conditions Eq. (5). Previous studies [2] have shown that a reasonable spatial resolution requires at least six mesh points per half roll (distance of $\pi$ ) or $\Delta r \leq \pi / 6 \approx 0.52$. For fixed $r_{1}$ and $r_{2}$, we therefore chose the spatial resolutions $N_{r}$ and $N_{\theta}$ to provide or to exceed this resolution in the radial and azimuthal directions. (Although the resolution is uniform in the $r$ and $\theta$ coordinates, in an annular cell geometry the field $\psi$ is approximated more accurately near the inner radius since the density of angular mesh points is higher there.) Many of our runs were tested at higher spatial and temporal resolutions (usually by a factor of 2 or 4 ) and our results were verified not to change with the higher resolution.

We used four different kinds of initial conditions $\psi_{0}(r, \theta)=\psi(t=0, r, \theta)$ to explore the dependence of the final pattern on the initial state,

$$
\begin{array}{ll}
\psi_{0,1}=a \cos \left(k_{\theta} \theta\right), & \psi_{0,2}=a \sin \left(k_{r} r+\phi\right), \\
\psi_{0,3}=a \sin \left(k_{x} r \cos \theta+\phi\right), & \psi_{0,4}=a \eta .
\end{array}
$$

These describe respectively radially oriented rolls $\left(\psi_{0,1}\right)$, azimuthally oriented (concentric) rolls $\left(\psi_{0,2}\right)$, straight rolls $\left(\psi_{0,3}\right)$, and random noise $\left(\psi_{0,4}\right)$. (We also occasionally added small-amplitude noise to the roll initial conditions to break possible symmetries.) The parameter $a$ denotes the initial amplitude (often chosen to be $0.1 \sqrt{\epsilon}$ ), the parameters $k_{\theta}, k_{r}$, and $k_{x}$ are specified wavenumbers, $\phi$ is some specified phase, and the $\eta$ are uniformly distributed random numbers in the interval $[-a, a]$. Although the second step Eq. (10) of the operating-splitting method automatically makes the solution $\psi(t+\Delta t)$ satisfy the discrete boundary conditions Eq. (5), we found that convergence to a smooth solution was enhanced if we forced the initial states Eq. (11) to satisfy the boundary conditions by setting the field $\psi_{0}$ to zero within a distance $\Delta r=\pi$ of the inner and outer radii.

The code then generated spatiotemporal fields $\psi_{i j k}$ on the polar $j k$-mesh at successive times $t_{i}=i \Delta t$. We visualized the fields with contour plots at positive and negative contours of magnitude $\pm(1 / 2) \max _{j, k}\left|\psi_{i j k}\right|$. We also plotted three scalar time series that proved useful when determining whether some state had become stationary. One time series was the local field value at the midpoint of the annulus at angle $\theta=0$

$$
s_{1}(t)=\psi\left(t, \frac{r_{1}+r_{2}}{2}, 0\right) .
$$

The second series was a global quantity, the spatially averaged mean-square field $\left\langle\psi^{2}\right\rangle(t)$

$$
s_{2}(t)=\frac{1}{A} \int_{r_{1}}^{r_{2}} d r \int_{0}^{2 \pi} d \theta r[\psi(t, r, \theta)]^{2}
$$

where $A=\pi\left(r_{2}^{2}-r_{1}^{2}\right)$ is the area of the annular domain. Near onset, one can show that Eq. (13) is linearly related to the global vertical heat transport (Nusselt number) across the fluid layer and so a plot of $s_{2}(t)$ indicates how heat transport depends on the spatiotemporal dynamics. As a third time series, we recorded the instantaneous value of the spatially averaged Lyapunov functional $\langle\mathcal{L}[\psi]\rangle$

$$
s_{3}(t)=\langle\mathcal{L}[\psi]\rangle(t)=\frac{1}{A} \mathcal{L}[\psi],
$$


where [3]:

$$
\mathcal{L}[\psi]=\int_{r_{1}}^{r_{2}} d r \int_{0}^{2 \pi} d \theta r\left[-\epsilon \psi^{2}+\frac{\epsilon}{2} \psi^{4}+\left\{\left(\nabla^{2}+1\right) \psi\right\}^{2}\right] .
$$

Because the mesh is uniform with respect to the $r$ and $\theta$ variables, the integrals in Eqs. (13) and (14) could be approximated with a simple two-dimensional version of the rectangle rule for approximating one-dimensional integrals [26]. The integrands were first approximated at the mesh points Eq. (6) using second-order accurate finite-difference approximations for the spatial derivatives, and then the mesh values were accumulated over all the mesh points. For many initial conditions, we verified that the time series $s_{3}(t)$ in Eq. (14) decreased monotonically with time provided that the space-time resolution was sufficiently fine, as should be the case for the analytical functional associated with Eq. (2) and with boundary conditions Eq. (5).

After observing many patterns and their time series, we chose empirically to define a pattern to be stationary if each of the two global time series Eq. (13) and Eq. (14) had converged to three significant digits. In fact, many of the patterns continued to evolve extremely slowly even after many horizontal diffusion times (since the phase of the stripe amplitude is diffusing in a large domain), and we saw changes in the fourth significant digit of the quantities $\left\langle\psi^{2}\right\rangle$ and $\langle\mathcal{L}\rangle$. However, when we integrated by a factor of ten longer for certain representative patterns (nearly $100 \Gamma^{2}$ in some cases), we found no significant changes in the patterns except for tiny translations or rotations of the overall roll structure. Although future calculations of stationary states may benefit by using more sophisticated algorithm such as a Newton method [26] applied to the time-independent form of Eq. (2) or by using an annular version of a recently developed fully-implicit method [13], the present results should provide a good starting point for comparing theory with experiment.

\section{RESULTS AND DISCUSSION}

\section{A. Overview}

In this section we summarize results of numerical integrations in annular domains of the Swift-Hohenberg equation Eq. (2) for different choices of the bifurcation parameter $\epsilon$, inner and outer radii $r_{1}$ and $r_{2}$, and initial conditions. Our results confirm some predictions of Cross [3] but also show reveal new patterns that arise when the aspect ratio Eq. (1) becomes sufficiently large. We also mention a few results for the Generalized Swift-Hohenberg equation which permits time-dependent nontransient states.

The space of parameters $\epsilon, r_{1}, r_{2}$, and initial data is too large to explore systematically so we let the predictions of Cross, as well as practical computational constraints guide us in our choice of parameters. Our interest was first to explore the region defined by the limits Eq. (1) for which the amplitude equation should give an accurate description of the dynamics. For the annular domain Eq. (3), Eq. (1) plus the assumption of Cross that the annular aspect ratio is not too large imply the following constraints

$$
r_{1} \sqrt{\epsilon} \gg 1, \quad\left(r_{2}-r_{1}\right) \sqrt{\epsilon} \gg 1, \quad \frac{r_{2}-r_{1}}{r_{1}} \ll 1,
$$

and we would like to satisfy these inequalities as best as possible. A second goal was to test the specific prediction of Cross [3] that a texture involving roll dislocations becomes more stable (has a lower Lyapunov functional value $\mathcal{L}$ in Eq. (14)) when the following inequality is first satisfied:

$$
\epsilon^{1 / 4} \geq \frac{r_{2}-r_{1}}{r_{1}}
$$

For fixed radii $r_{1}$ and $r_{2}$, Eq. (17) can be tested by making runs for $\epsilon$ above and below the ratio $\left[\left(r_{2}-r_{1}\right) / r_{1}\right]^{4}$ for the four initial conditions Eq. (11), followed by comparing the average value of the Lyapunov functional $\langle\mathcal{L}\rangle$ for the final stationary states.

Another constraint on the choice of parameters $\epsilon, r_{1}$, and $r_{2}$ for an annular domain comes from the Busse stability balloon for the Swift-Hohenberg equation (see Fig. 31 in Ref. 22). For locally parallel rolls that are normal to the inner and outer walls of an annulus, one might expect a pattern change when the local wavenumber $q(r)$ as a function of radius crosses a linear stability boundary. For example, if the local wave number $q$ near a fixed outer radius $r_{2}$ is as small as possible consistent with the zig-zag stable region $q \geq 1$, then the local wave number $q\left(r_{1}\right)$ enters the Eckhaus unstable region $q \geq 1+\sqrt{\epsilon / 12}$ when 


$$
r_{1} \leq \frac{r_{2}}{1+\sqrt{\epsilon / 12}}
$$

For $\epsilon=0.1$ and fixed $r_{2}=80$ (two parameters used in the runs below), the local wavenumber $q\left(r_{1}\right)$ becomes Eckhaus unstable when $r_{1} \leq 73.3$. The corresponding aspect ratio $\Gamma \approx 2.1$ is so small that the critical value $\epsilon_{c}$ for the instability of the zero-field state in a finite system exceeds $\epsilon=0.1$, i.e., as soon as a finite amplitude radial stripe pattern can form, it is already Eckhaus unstable. A larger outer radius $r_{2}$ would therefore be needed to test the implications of Eq. (18).

Given the above constraints and our computational resources, we chose to fix the outer radius at the value $r_{2}=80$ (about 25 half-rolls) and study patterns with a decreasing sequence of inner radii, from $r_{1}=60$ to $r_{1}=10$ in steps of 10. This corresponds to increasing the aspect ratio $\Gamma$ from 6.4 to 22.3 in steps of $\Delta \Gamma=3.2$. Eq. (16) then implies that we can make comparisons with Cross's prediction Eq. (17) for $\Gamma \leq 10$. For the different choices of $r_{1}$ for fixed $r_{2}$, we studied just two values of the bifurcation parameter, $\epsilon=0.1$ and $\epsilon=0.5$. Earlier calculations of the Swift-Hohenberg equation in rectangular domains [2] show that barriers to dislocation motion appear for $\epsilon$ larger than about 0.5 so these two values probe the "near onset" and "not so close to onset" regimes.

\section{B. Stationary Patterns}

We begin our discussion of results with Fig. 1, which shows two asymptotic stationary patterns near onset $(\epsilon=0.1)$ obtained by integrating the Swift-Hohenberg equation for more than 15 horizontal diffusion times (units of $\tau_{h}=\Gamma^{2}$ ) in a small aspect ratio $\Gamma \simeq 6.4$ annular domain of outer radius $r_{2}=80$. The initial conditions for panels (a) and (b) were respectively radially oriented rolls and straight rolls $\left(\psi_{0,1}\right.$ and $\psi_{0,3}$ in Eq. (11)). (Random initial conditions lead to the same kind of state so a pattern with dislocations seems to be the most accessible basin of attraction.) The asymptotic patterns in both cases consist of approximately straight radially oriented rolls disrupted by four to six dislocations. The states in (a) and (b) have an identical average Lyapunov functional $\langle\mathcal{L}\rangle=3.40 \times 10^{-3}$ to three significant digits and so are extremely close in "energy". Initial conditions consisting of concentric rolls of high symmetry and a little noise evolve into nonlinear stationary concentric rolls which therefore constitute another basin of attraction, although a small one. The average value of the Lyapunov function $\langle\mathcal{L}\rangle=0.0819$ is higher than that for any of the patterns with dislocations so the concentric rolls are not preferred.

The value $\epsilon=0.1 \mathrm{in} \mathrm{Fig.} \mathrm{1} 1$ substantially exceeds the value $\left[\left(r_{2}-r_{1}\right) / r_{1}\right]^{4} \approx 0.012$ predicted by the criterion Eq. (17) with $r_{2}=80, r_{1}=60$ and so Fig. 1 is consistent with Cross's prediction that states with dislocations are preferred in that they have a lower average Lyapunov functional. In fact, because the threshold $\epsilon \approx 0.012$ defined by Eq. (17) is smaller than the critical value $\epsilon_{c}$ for the onset of convection in such a small aspect ratio cell, we could not find a pattern of straight rolls for these same parameters to compare Lyapunov functional values. For the larger value of $\epsilon=0.5$, which also satisfies Eq. (17), asymptotic patterns similar to Fig. 1 are observed for initial conditions consisting of radially oriented rolls or of random values. These patterns further from onset have an average Lyapunov functional and average square value of $\langle\mathcal{L}\rangle=-0.0875$ and $\left\langle\psi^{2}\right\rangle=2.79 \times 10^{-5}$. But for an initial condition consisting of straight rolls at the critical wave number $q=1$, Fig. 2 shows that a dislocation-free pattern of approximately straight rolls can occur, although the stripes are only approximately straight where they are nearly parallel to the inner and outer boundaries. This pattern has average values of $\langle\mathcal{L}\rangle=0.0819$ and $\left\langle\psi^{2}\right\rangle=2.74 \times 10^{-4}$ and so is not preferred, again in accordance with the criterion Eq. (17).

We now increase the aspect ratio from $\Gamma=6.4$ to 12.8 by decreasing the inner radius from $r_{1}=60$ to 40 for fixed $r_{2}=80$. Cross's criterion Eq. (17) for a texture with dislocations to be preferred is now no longer expected to hold since the annulus is many rolls wide and more complex textures can be expected. Fig. 3 shows two examples of the approximately stationary patterns observed after long times near onset $(\epsilon=0.1)$, starting from two different initial conditions, radially oriented rolls in (a) and straight rolls in (b). The patterns have some similarities to Fig. 1 in that the rolls are largely radially oriented but they now bend at a substantial angle as they traverse the outer to inner boundaries and a novel defect structure is observed in the form of four or five radially-oriented grain boundaries. These and other runs suggest that there are many different stationary states possible for the specified parameter values, all differing slightly in the position and number of the grain boundaries and possibly with the presence of a few other defects. For example, in addition to four grain boundaries Fig. 3(b) shows two dislocations near the top and bottom of the inner boundary. According to the values of $\mathcal{L}$, Fig. 3(a) is slightly preferred over (b) but the lack of symmetry suggests that neither is the global minimum for Eq. (15).

For this same $\Gamma=12.8$ cell, we carried out several runs further from onset for the value $\epsilon=0.5$. Straight roll initial states lead to a straight-roll stationary state (not shown) similar to Fig. 2 with average values of $\langle\mathcal{L}\rangle=-0.176$ and $\left\langle\psi^{2}\right\rangle=3.26 \times 10^{-5}$. Radially-oriented and random initial conditions lead to patterns similar to Fig. 1 1 but with many more defects (with values $\langle\mathcal{L}\rangle=-0.176$ and $\left\langle\psi^{2}\right\rangle=3.19 \times 10^{-5}$ ). Although the straight-roll state has a 
lower average Lyapunov value, it is not accessible from typical initial conditions because of the barriers to dislocation motion.

Fig. 1 shows two stationary patterns near onset $(\epsilon=0.1)$ in a still larger $\Gamma=19.2$ cell, while Fig. . stationarity of such patterns by showing how the time series for the average Lyapunov functional $\langle\mathcal{L}\rangle$ and mean square field $\left\langle\psi^{2}\right\rangle$ have converged to constant values. A new feature in Fig. A(a) is the appearance of a focus singularity for the first time (near the upper right side of the annulus). Foci are a common feature in large cylindrical and rectangular patterns near onset but evidently don't occur in annular domains until the aspect ratio is sufficiently large. The stationary state Fig. G(b) arises from straight-roll initial conditions. Again there is a new feature with this larger aspect ratio in the form of grain boundaries next to the top and bottom outer boundary. Such grain boundaries are a common feature in large rectangular cells near onset (for example, see Fig. 9 of Ref. 21]) since rolls parallel to a sidewall are known to be unstable near onset to transverse rolls. The defected and straight roll patterns in Figs. 河(a) and (b) have identical average Lyapunov functionals to three significant digits and so are equally stable. However, the straight roll state empirically has a tiny basin of attraction and so is not accessible for most initial states.

We conclude this subsection with Fig. 6, which shows two representative patterns in the $\Gamma=19.2$ cell further from onset for $\epsilon=0.5$. Fig. 6 (a) grew out of straight roll initial conditions and is a nearly perfect array of stripes; the instability near sidewalls towards transverse rolls does not occur at this value of $\epsilon$. Fig. 6(b) shows a state that grew out of small amplitude random initial conditions. The highly disordered lamellar-like pattern is similar to that observed for the Swift-Hohenberg equation in a large rectangular cell for $\epsilon=0.5$ [2] and is a consequence of larger barriers to the motion of dislocations. Such frozen disordered states have not been observed experimentally and are likely an artifact of the Swift-Hohenberg equation, which is most physically relevant to Rayleigh-Bénard convection in the limit $\epsilon \rightarrow 0$ and large Prandtl number.

\section{Dynamical States of the Generalized Swift-Hohenberg Equation}

Convection experiments close to onset of room-temperature pressurized gases (Prandtl number $\approx 1$ ) have revealed many different sustained time-dependent states of which spiral defect chaos (SDC) 18, 27 31 has been perhaps the most intriguing and thoroughly studied. Several experimental features of SDC remain poorly understood, e.g., why the state occurs in the first place, why its statistical properties are insensitive to the aspect ratio of a cell (above some minimum value of $\Gamma$ ), and why, to the contrary, the critical value $\epsilon_{\mathrm{SDC}}$ above which SDC is first observed (for a fixed experimental cell) is sensitive to the size and geometry of the convection cell.

To determine whether SDC may be modified by an annular geometry and whether the wave number frustation of an annular cell may itself be a source of sustained dynamics, we have carried out a few exploratory calculations with a Generalized Swift-Hohenberg model [11, 15] for which prior numerical calculations in large square periodic domains have produced spiral-defect-chaos like states [18, 29, 32]. We used a model of the form

$$
\begin{aligned}
\partial_{t} \psi & =\left[\epsilon-\left(\nabla^{2}+1\right)^{2}\right] \psi-\psi^{3}-g(\mathbf{U} \cdot \nabla) \psi, \\
\left(-\eta \nabla^{2}+c^{2}\right) \nabla^{2} \zeta & =\hat{z} \cdot\left[\nabla\left(\nabla^{2} \psi\right) \times \nabla \psi\right], \\
\mathbf{U} & =\nabla \times(\zeta \hat{z}),
\end{aligned}
$$

with boundary conditions Eq. (5) for the field $\psi$ and boundary conditions

$$
\zeta\left(r_{1}\right)=\zeta\left(r_{2}\right)=0,\left.\quad \partial_{r} \zeta\right|_{r_{1}}=\left.\partial_{r} \zeta\right|_{r_{2}}=0,
$$

for the vorticity potential field $\zeta(t, r, \theta)$. Because Eq. (20) is linear in $\zeta$ and involves again a constant-coefficient generalized biharmonic operator, it was straightforward to modify the numerical algorithm of Section II to integrate Eqs. (19)- (21) in time.

For all of our runs, we used the parameters of Ref. [29]

$$
\epsilon=0.5, \quad \eta=1, \quad g=50, \quad c^{2}=2,
$$

for which long-lived spiral-defect-chaos-like states were observed with periodic boundary conditions. We then fixed the outer radius to be $r_{2}=80$ and made several runs, each starting with random initial conditions, for values of the inner radii from $r_{1}=60$ to $r_{1}=10$ decreasing in steps of -10 . (This corresponds to aspect ratios between 6.4 and 22.3 in steps of $\Delta \Gamma \approx-3.2$.) For $\Gamma<13$, we found that all states eventually became stationary (using the time series of $\left\langle\psi^{2}\right\rangle$ as the criterion) with the state $\Gamma=12.7$ taking the long time of $43 \tau_{h}$ to become time independent. For $\Gamma>13$, the states were still dynamic up to our longest integration times of $50 \tau_{h}$ although there was evidence both visually and from time series that the states were coarsening and slowly evolving to a state other than SDC. 
A representative example of the spatiotemporal dynamics of the GSH model Eqs. (19)- (21) in a large aspect ratio $\Gamma=22.3$ annular cell is shown in Fig. 6 . The contour plots show the spatial structure of the field $\psi$ at times $t=4,12$, 18 , and $24 \tau_{h}$. At earlier times (panel a), there are a few spirals but these eventually annihilate leaving a pattern that is more slowly evolving, and with nearly half the pattern consisting of foci. Two associated time series are shown in Fig. 8. They suggest that the dynamics is more complex for about 10 horizontal diffusion times, then becomes slower and simpler, unlike the experimentally observed SDC state. The approximately periodic behavior of the midpoint field value in Fig. \& $\mathrm{b}$ for $t>15 \tau_{h}$ is likely caused by a slow translation of stripes past the observation point and does not correspond to a true time-periodic behavior. These time series suggest that the pattern may eventually become time independent.

Because the approximations relating the GSH model to the Boussinesq equations are less well justified than those that relate the Swift-Hohneberg model near onset, especially concerning sustained chaotic states, it would be interesting to continue these calculations not with further integrations of the GSH model but with integrations of the three-dimensional Boussinesq equations in an annular domain, and to test the various calculations with experiments in large-aspect-ratio annular domains.

\section{CONCLUSIONS}

The previous sections may be summarized as follows. We have developed and applied a new computer code to study pattern formation formation near onset of the Swift-Hohenberg model in annular domains of varying aspect ratios. As pointed out by Cross [3], an annular geometry is interesting because there is an inherent conflict near onset between the tendency of rolls to be normal to the sidewalls and the tendency for the rolls to be straight in the bulk. We have investigated Cross's predictions for annular cells of varying aspect ratio $6.4 \leq \Gamma \leq 22.3$, near onset with $\epsilon=0.1$, and further from onset with $\epsilon=0.5$.

For an annular domain of rather small aspect ratio $(\Gamma<5)$, the predictions of Cross hold and stationary states with dislocations are preferred (have a lower Lyapunov functional density) compared to straight rolls. As the aspect ratio $\Gamma$ becomes larger, new patterns are observed that are characterized by radially oriented grain boundaries. In nearly all cases, for a given geometry and reduced Rayleigh number $\epsilon$, many different stationary states are possible, usually differing only slightly in the value of the Lyapunov density or mean square field (Nusselt number). The pattern that produces the global minimum of $\mathcal{L}$ for given parameters values $\epsilon, r_{1}$, and $r_{2}$ is not known but we conjecture to be a highly symmetric arrangement of defects. Straight-roll patterns are observed only when starting from straight-roll initial conditions. Such patterns can have a lower Lyapunov density than the patterns with radially oriented rolls but evidently have such a small basin of attraction that they are not observed starting from most initial conditions.

We also explored the possibility of sustained time-dependent states using a Generalized Swift-Hohenberg model, for parameters such that a long-lived spiral defect state is observed in a large aspect ratio periodic square [29]. However our admittedly incomplete study revealed only long-lived complex spatiotemporal transients. Further analysis, preferably by experiment or by integrations of the Boussinesq equations, will be needed to determine the effect of an annular domain on time-dependent states.

We hope that the above results will stimulate experiments to test the specific calculations reported here, especially in large Prandtl number fluids near onset for which the Swift-Hohenberg equation should provide a reasonable description.

\section{ACKNOWLEDGEMENTS}

We would like to thank Michael Cross for helpful discussions and NSF grant DMS-9722814 and DOE grant DEFT02-98ER14892 for supporting this research.

FIG. 1. Stationary numerical solutions of the Swift-Hohenberg equation Eq. (2) with boundary conditions Eq. (50) in an annular domain of outer radius $r_{2}=80$, inner radius $r_{1}=60$, aspect ratio $\Gamma \simeq 6.4$, and bifurcation parameter $\epsilon=0.1$. Part (a): A pattern at time $t=17 \tau_{h}$ (units of horizontal diffusion times $\tau_{h}$ ) that grew out of the initial state $\psi_{0}=0.3 \cos (100 \theta)$. Part (b): a pattern at time $19 \tau_{h}$ that grew out of a straight-roll initial state $\psi_{0}=0.3 \cos (r \cos (\theta))$. For both patterns, $\langle\mathcal{L}\rangle[\psi]=3.40 \times 10^{-3}$ and $\left\langle\psi^{2}\right\rangle=4.43 \times 10^{-6}$. The spatiotemporal resolutions were $N_{r}=66, N_{\theta}=1024$, and $\Delta t=0.5$.

FIG. 2. Stationary pattern at time $t=25 \tau_{h}$ starting from straight roll initial conditions $\psi_{0}=0.3 \cos (r \cos (\theta))$ for the same physical and numerical parameters as in Fig. 1 but with a larger value $\epsilon=0.5$. Now $\langle\mathcal{L}\rangle=0.0819$ and $\left\langle\psi^{2}\right\rangle=2.74 \times 10^{-5}$. 
FIG. 3. Two stationary patterns in a larger annular domain for parameters $\epsilon=0.1, r_{1}=40, r_{2}=80, \Gamma \approx 12.7, \Delta t=0.5$, $N_{r}=130$ and $N_{\theta}=1024$. (a): An approximately stationary pattern obtained at time $t=19 \tau_{h}$ starting from azimuthally oriented rolls, with values $\langle\mathcal{L}\rangle=-4.50 \times 10^{3}$ and $\left\langle\psi^{2}\right\rangle=3.32 \times 10^{-6}$. (b): An approximately stationary pattern obtained at time $t=22 \tau_{h}$ starting from straight roll initial conditions, with $\langle\mathcal{L}\rangle=-4.45 \times 10^{-3}$ and $\left\langle\psi^{2}\right\rangle=3.38 \times 10^{-6}$. Both patterns show new defects consisting of radially oriented grain boundaries.

FIG. 4. Two stationary patterns in an annular domain for parameters $\epsilon=0.1, r_{1}=20, r_{2}=80, \Gamma \approx 19.2, \Delta t=0.5$, $N_{r}=258$ and $N_{\theta}=512$. (a): An approximately stationary pattern obtained at time $t=22 \tau_{h}$ starting from radially-oriented rolls. (b): An approximately stationary pattern obtained at time $t=22 \tau_{h}$ starting from straight roll initial conditions. These and similar patterns obtained starting from random initial conditions all have identical average Lyapunov functionals and square values to three significant digits, with respectively $\langle\mathcal{L}\rangle=-4.86 \times 10^{-3}$ and $\left\langle\psi^{2}\right\rangle=2.88 \times 10^{-6}$.

FIG. 5. Time series of the spatially averaged Lypuanov functional $\langle\mathcal{L}\rangle(t)$ (Eq. (14)) and mean-square value $\left\langle\psi^{2}\right\rangle(t)($ Eq. $(13))$ starting from low-amplitude random initial conditions for the parameters of Fig. 4 . The states are accurately stationary for $t>19 \tau_{h}$, although it is rather interesting that the two time series do not become stationary at the same time.

FIG. 6. Two stationary patterns in an annular domain for parameters $\epsilon=0.5, r_{1}=20, r_{2}=80, \Gamma \approx 19.2, \Delta t=0.5$, $N_{r}=258$ and $N_{\theta}=512$. (a): An approximately stationary pattern obtained at time $t=2 \tau_{h}$ starting from straight rolls, with values $\mathcal{L}=-0.110$ and $\left\langle\psi^{2}\right\rangle=1.59 \times 10^{-5}$. An essentially perfect straight roll state is attained. (b): An approximately stationary pattern obtained at time $t=8 \tau_{h}$ starting from small-amplitude random initial conditions. Although there are many defects, the rolls are still oriented normal to the boundaries.

FIG. 7. Contour plots of the field $\psi(t, r, \theta)$ at times $t=6,12,18$, and $24 \tau_{h}$ from an integration of the Generalized-Swift-Hohenberg equations Eqs. (19)- (21) with boundary conditions Eqs. (5) and (22) in an annular domain with $r_{2}=80$, $r_{1}=10, \Gamma \approx 22.3$ and starting from small-amplitude random initial conditions. The parameters used were $\epsilon=0.5, \eta=1$, $c^{2}=2, g=50, N_{r}=514, N_{\theta}=1024$, and $\Delta t=0.125$. The state is time dependent out to $27 \tau_{h}$, the longest time observed.

FIG. 8. Time series of (a), the mean square field $\left\langle\psi^{2}\right\rangle$ and of (b), the midpoint value Eq. (12) for the time dependent solution of the GSH equations shown in Fig. 7. The dynamics is becoming slower and simpler over time.

* $\quad$ E-mail correspondence should be sent to hsg@phy.duke.edu.

$\dagger \quad$ Also Duke University's Center for Nonlinear and Complex Systems.

[1] J. Swift and P. C. Hohenberg, Phys. Rev. A 15, 319 (1977).

[2] H. S. Greenside and W. M. Coughran, Jr., Phys. Rev. A 30, 398 (1984).

[3] M. C. Cross, Phys. Rev. A 25, 1065 (1982).

[4] M. S. Heutmaker and J. P. Gollub, Phys. Rev. A 35, 242 (1987).

[5] V. Croquette, Contemporary Physics 30, 113 (1989).

[6] E. Bodenschatz, J. R. de Bruyn, G. Ahlers, and D. S. Cannell, Phys. Rev. Lett 67, 3078 (1991).

[7] M. C. Cross and P. C. Hohenberg, Rev. Mod. Phys. 65, 851 (1993).

[8] F. H. Busse, Rep. Prog. Phys. 41, 1929 (1978).

[9] J. P. Gollub and A. R. McCarriar, Phys. Rev. A 26, 3470 (1982).

[10] D. A. Egolf, I. V. Melnikov, and E. Bodenschatz, Phys. Rev. Lett. 80, 3228 (1998).

[11] H. S. Greenside and M. C. Cross, Phys. Rev. A 31, 2492 (1985).

[12] Y. Tu and M. C. Cross, Phys. Rev. Lett. 69, 2515 (1992).

[13] M. Louie, Ph.D. thesis, California Institute of Technology, 2001.

[14] B. Sensoy, "Pattern Formation of a Convecting Fluid in an Annular Domain" undergraduate senior thesis, Duke University, 1999. Available at http://www.phy.duke.edu/undergraduate/thesis/sensoy/.

[15] P. Manneville, J. Physique 44, 759 (1983).

[16] H. S. Greenside, M. C. Cross, and W. M. Coughran, Jr., Phys. Rev. Lett. 60, 2269 (1988). 
[17] H.-W. Xi, J. D. Gunton, and J. Viñals, Phys. Rev. Lett. 71, 2030 (1993).

[18] M. Cross, Physica D 97, 65 (1996).

[19] R. Schmitz, W. Zimmermann, and W. Pesch, preprint,http://www.phy.uni-bayreuth.de/ btp406/publis/comment.ps.gz (unpublished).

[20] M. C. Strain and H. S. Greenside, Phys. Rev. Lett. 80, 2307 (1998).

[21] P. Bjørstad, in Notes on Numerical Fluid Mechanics, edited by W. Hackbusch (Vieweg, Braunschweig, 1984), Vol. 10, pp. $1-10$.

[22] J. C. Strikwerda, Finite Difference Schemes and Partial Differential Equations (Wadsworth \& Brookes/Cole, Pacific Grove, California, 1989).

[23] P. E. Bjørstad, SIAM J. Num. Anal. 20, 59 (193).

[24] P. E. Bjørstad et al., in Elliptic Problem Solvers II, edited by G. Birkhoff and A. Schoenstadt (Academic Press, New York, 1984), Chap. V, pp. 531-543.

[25] Numerical network library (netlib), Web site http://www.netlib.org, 2000.

[26] D. Kincaid and W. Cheney, Numerical Analysis, 2nd ed. (Brooks/Cole, Pacific Grove, California, 1996).

[27] S. W. Morris, E. Bodenschatz, D. S. Cannell, and G. Ahlers, Phys. Rev. Lett. 71, 2026 (1993).

[28] W. Decker, W. Pesch, and A. Weber, Phys. Rev. Lett. 73, 648 (1994).

[29] H. Xi and J. D. Gunton, Phys. Rev. E 52, 4963 (1995).

[30] S. Morris, E. Bodenschatz, D. Cannell, and G. Ahlers, Physica D 97, 164 (1996).

[31] R. V. Cakmur, D. A. Egolf, B. B. Plapp, and E. Bodenschatz, Phys. Rev. Lett. 79, 1853 (1997).

[32] Werner Pesch and collaborators (private communication) have used a spectral code with fine space-time resolution to analyze spiral defect chaos states of the Generalized Swift-Hohenberg model in a large periodic square domain and found an extremely long-lived transient that coarsens to a chaotic state with just a few spirals. Thus there remains some controversy whether a sustained spiral defect state truly exists in the GSH model as suggested by previous work [18, 29]. 

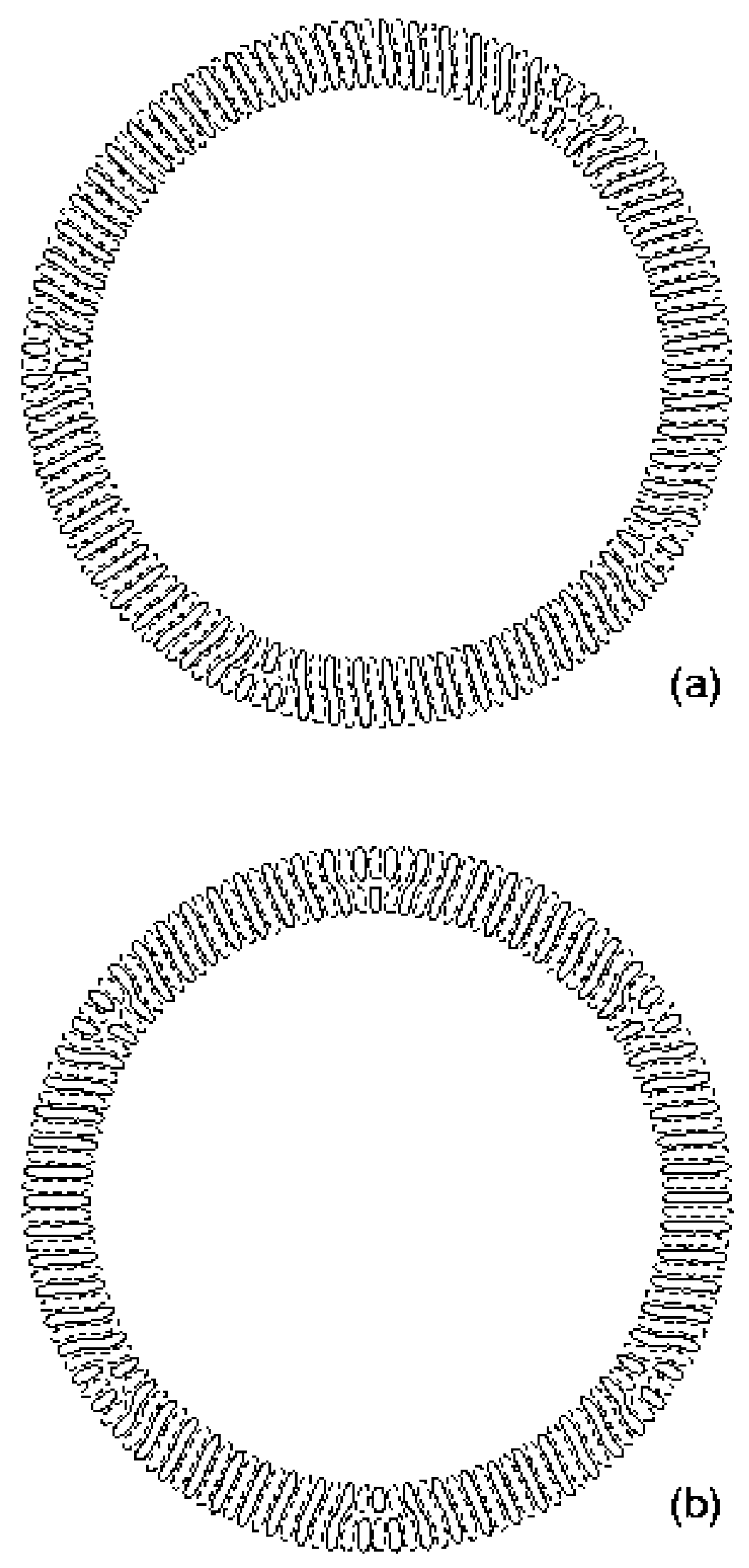

Figure 1. 


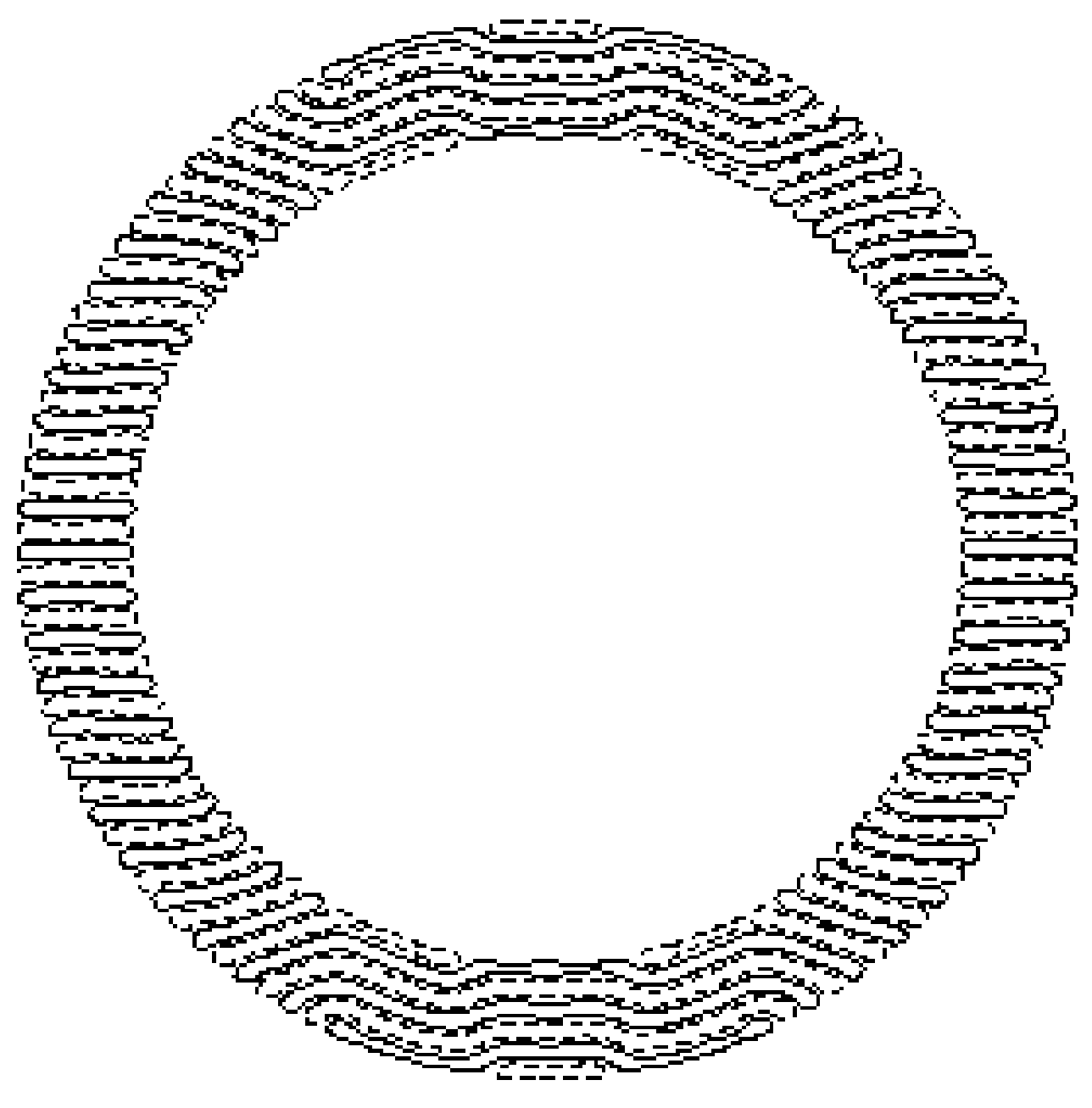

Figure 2. 

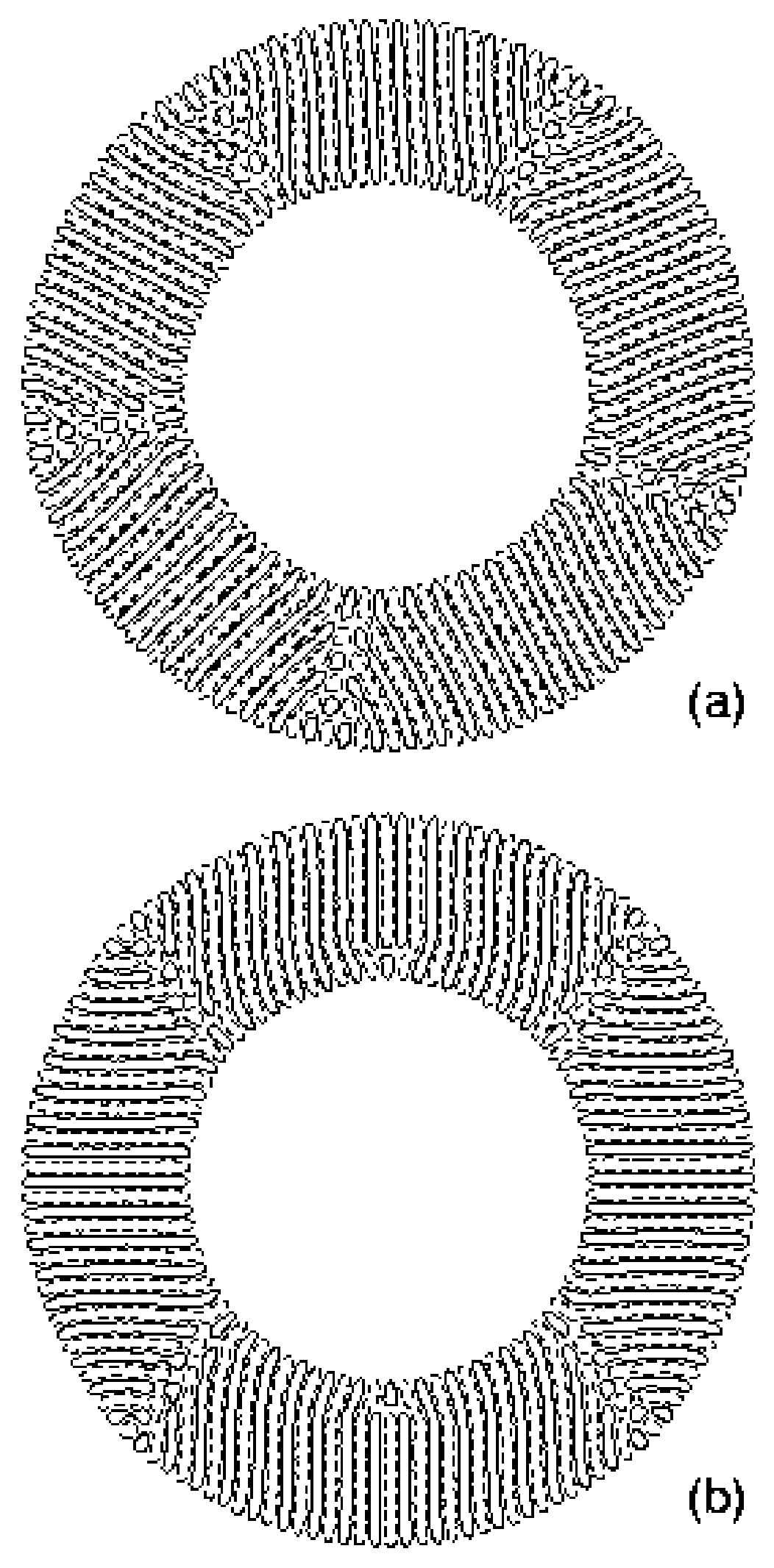

Figure 3. 

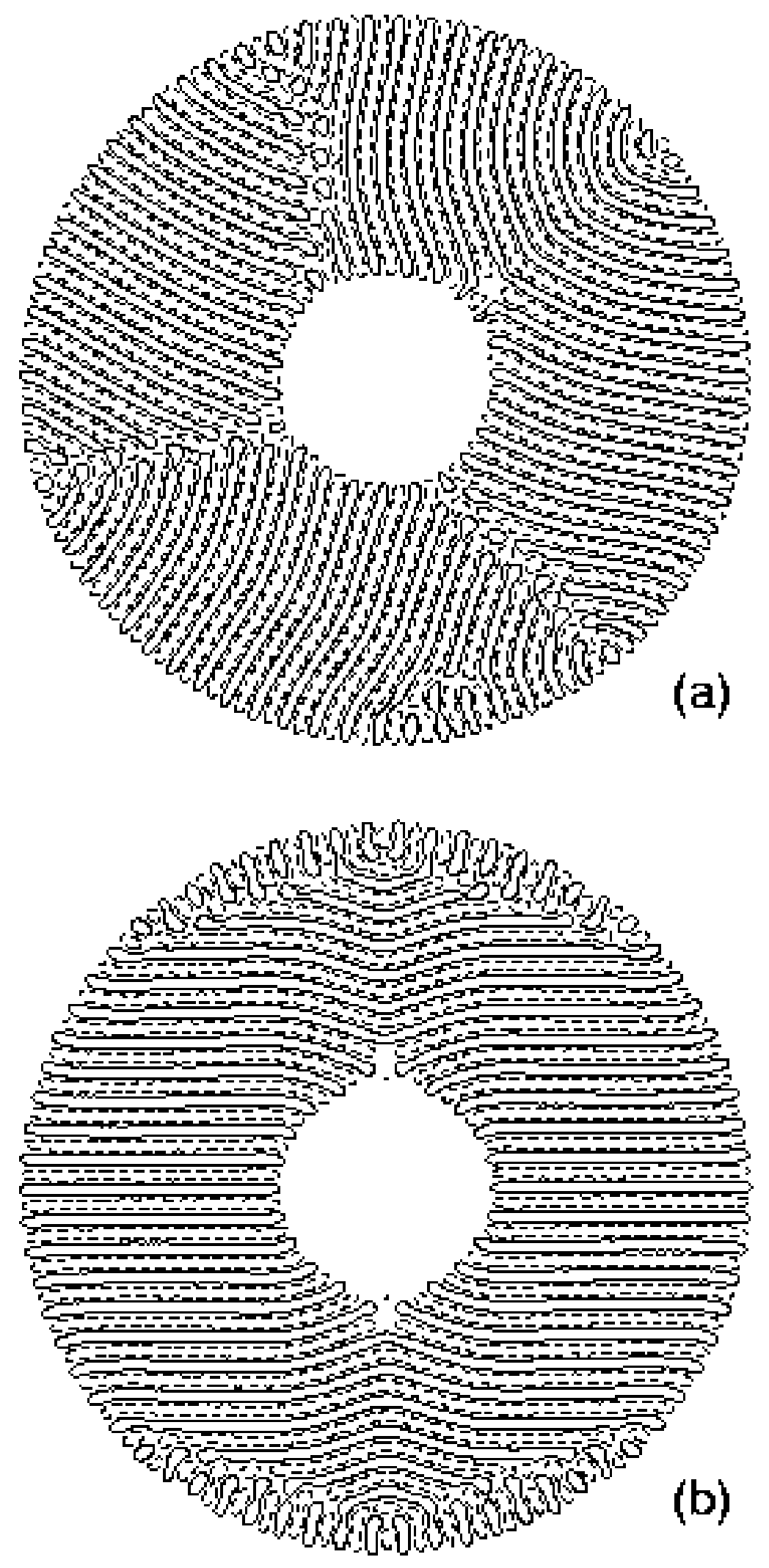

Figure 4 . 

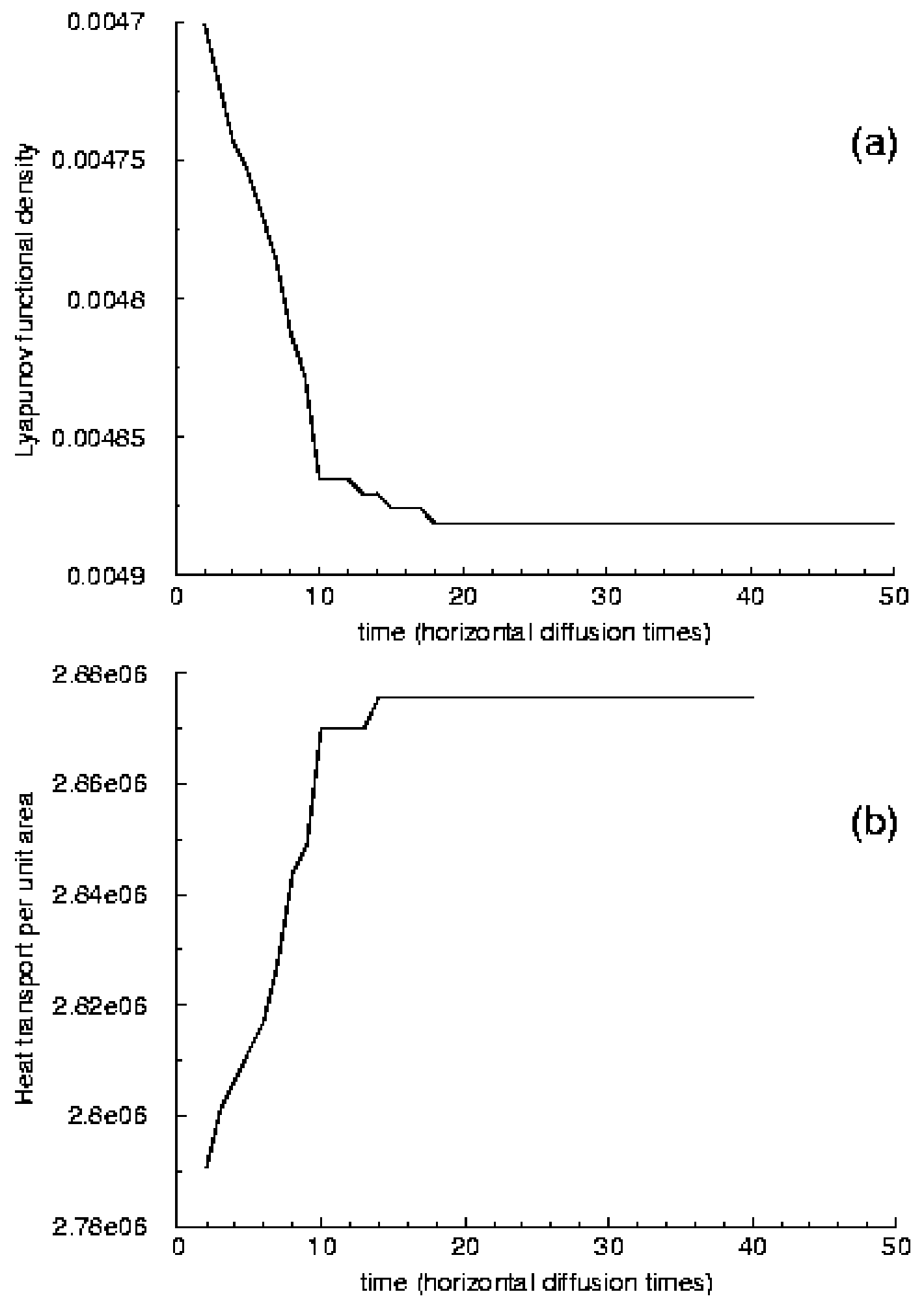

Figure 5. 

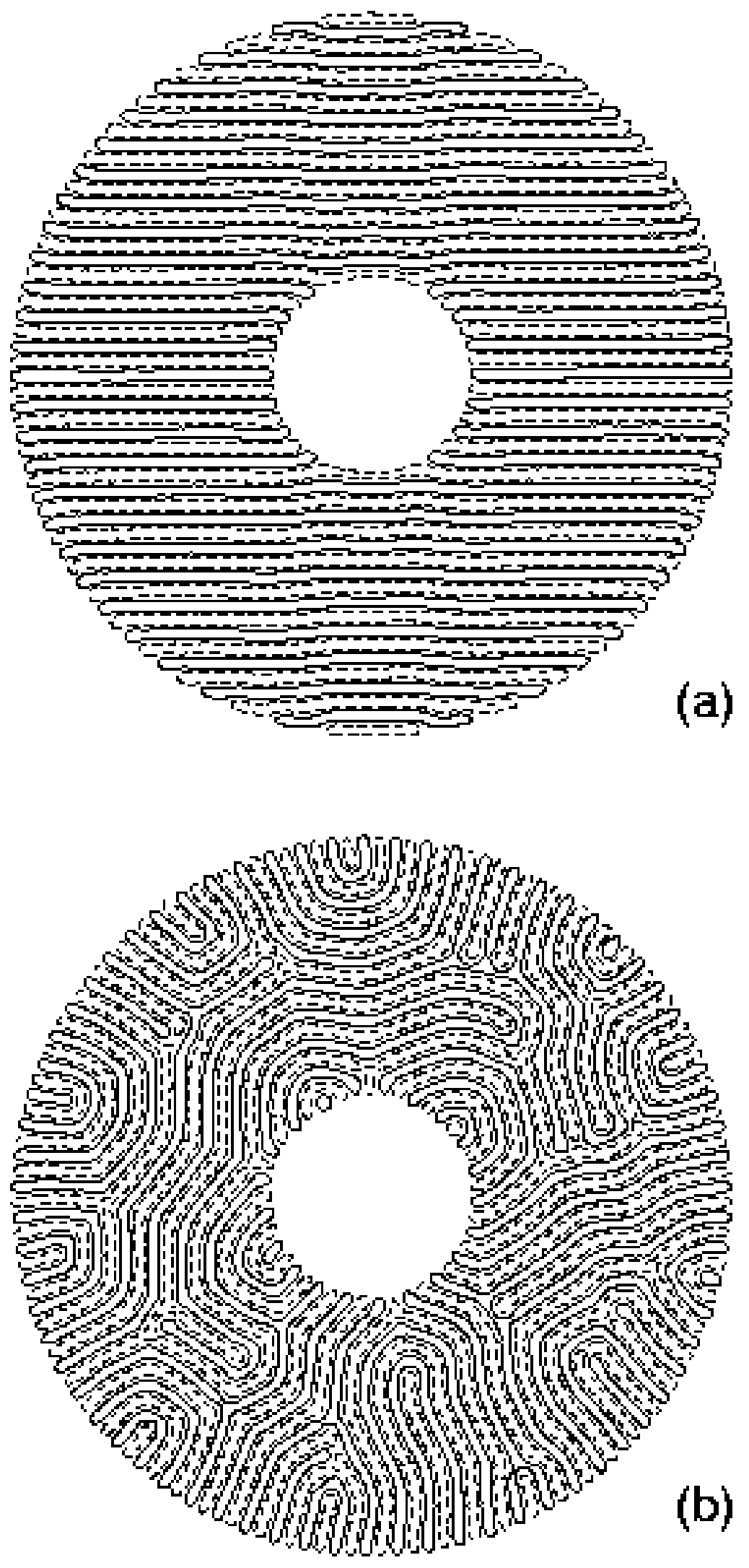

Figure 6 

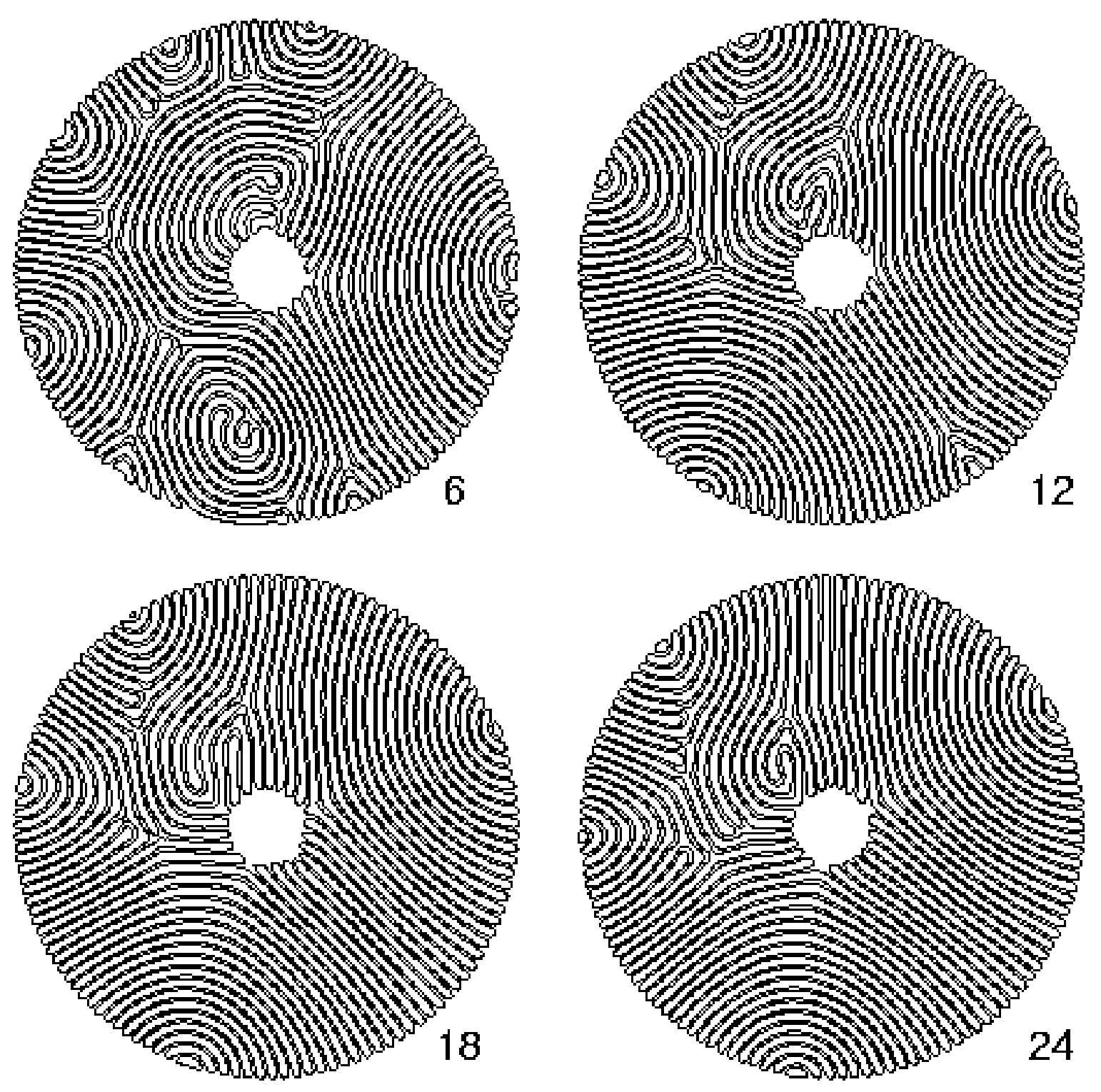

Figure 7. 

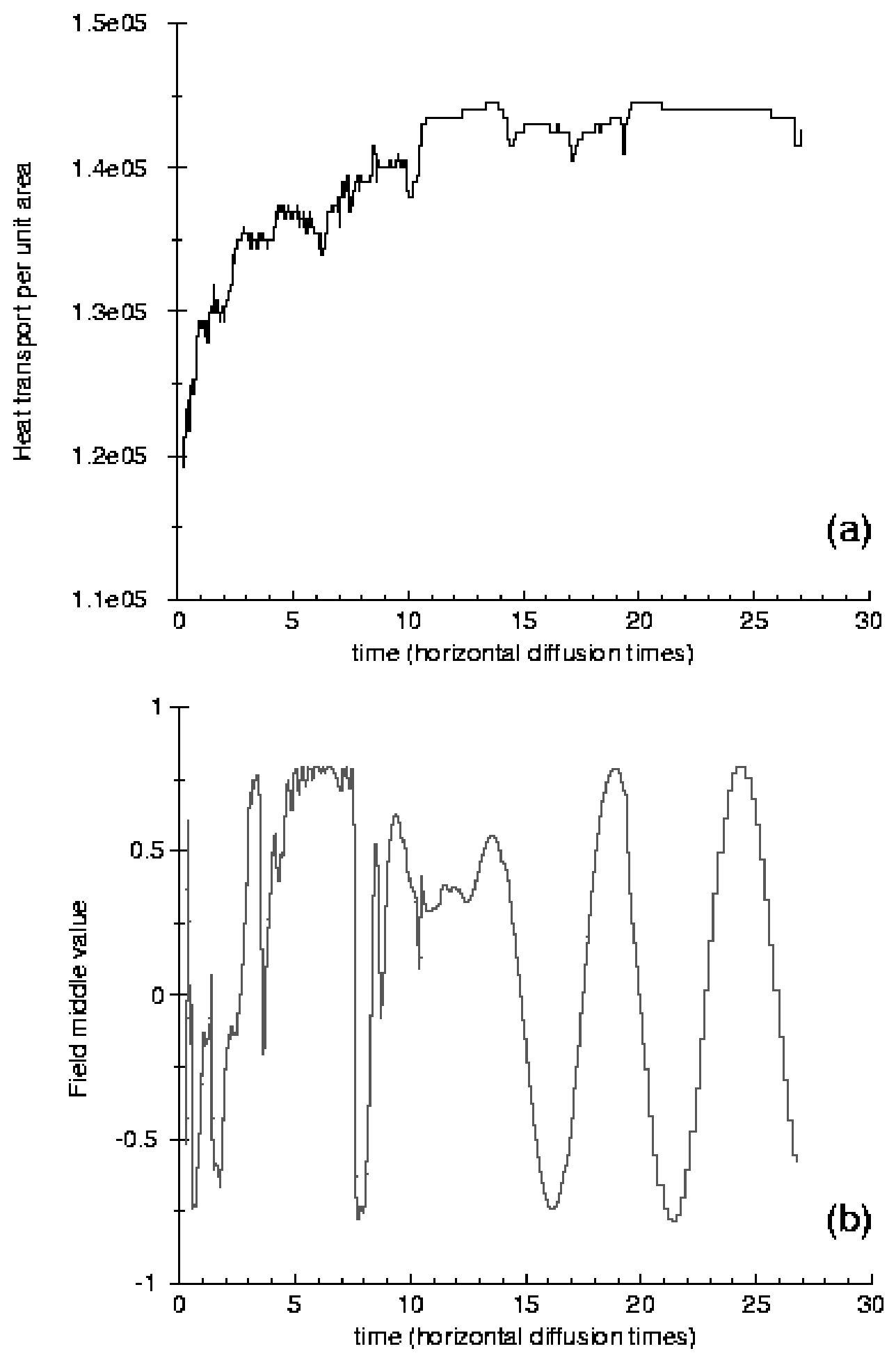

Figure 8. 